\title{
Metabolic activity assessment of cardiac angiosarcoma by 18PDCG PET-CT
}

Marek Bilski ${ }^{1,3}$, Grzegorz Kamiński', Mirosław Dziuk ${ }^{2,3}$

'Department of Endocrinology and Isotope Therapy, Military Institute of Medicine, Warsaw, Poland

${ }^{2}$ Department of Nuclear Medicine, Military Institute of Medicine,

Warsaw, Poland

${ }^{3}$ Masovian PET/CT Centre, Euromedic Diagnostics, Warsaw, Poland

[Received 2 || 2012; Accepted 16 || 2012]

\section{Introduction}

Angiosarcoma is a neoplasm of an endothelial origin, which could arise from blood or lymphatic vessels, respectively hemangiosarcoma or lymphosarcoma. They account for only $0.1 \%$ of adult malignancies and represent $1-2 \%$ of all soft tissue sarcomas in humans [1]. Most tumours are malignant and readily give distant metastases because they are composed of rapidly proliferating and extensively infiltrating anaplastic cells. They can occur in any organ of the body and the most common form is a cutaneous angiosarcoma [2]. We report a PET-CT study of a rare case of cardiac angiosarcoma, which was initially misdiagnosed as heart inflammation.

\section{Case report}

The 30 year old male with heart angiosarcoma was referred for PET-CT with 18FDG study. The patient had had no significant medical history and at the beginning was hospitalised because of a continuos pain in the pericardial region, initially misdiagnosed as pericarditis. The performed CAT scan showed pathological mass in the right atrium. The patient was subsequently subjected

Correspondence to: Marek Bilski

Department of Endocrinology and Isotope Therapy,

Military Institute of Medicine

128 Szaserow St., 04-141 Warsaw, Poland

Tel: +486817123, fax: +486817114

e-mail: mbilski@wim.mil.pl to a surgical tumour resection but it occurred inoperable. The histo-pathology examination performed after biopsy revealed a well differentiated cardiac angiosarcoma with an adhesion molecule CD31 and a CD34 expression. After three courses of adjuvant chemotherapy consisted of Adriamycin (20 mg), Dacarbazine (200 mg) and Cyclophosphamide (100 mg) the patient was referred to the PET-CT Centre for treatment efficiency assessment [3].

The PET-CT study was performed according to a standard protocol: 2.5 minutes acquisition per bed position (GE Discovery ${ }^{\mathrm{TM}}$ STE PET-CT scanner), 45 minutes after $5 \mathrm{MBq}$ per kilogram body mass 18FDG injection proceeded by a six hours fasting period [4]. It revealed an intensive metabolic activity (SUVmax $=10.3)$ in the tumour mass surrounding right atrium cavity (Figure 1). The maximum thickness of the neoplasmatically-infiltrated atrium located in its basal part amounted to $30 \mathrm{~mm}$. Concomitant pericardial effusion was noticed. In addition, multiple metabolically active (up to SUVmax =9.2) metastases were found in the skeleton (not visible on the CT scan) with no evidence of pulmonary or other soft tissue involvement (Figure 2).

\section{Discussion}

There are only few case reports concerning PET-CT with 18FDG in cardiac angiosarcoma. They are proving its usefulness in assessing malignant potential of the tumour mass and exploration for distant metastases in primary diagnosis together with metabolic response to and detection of local recurrence after applied therapy [5-8]. In the presented case PET-CT with 18FDG depicted persisted high metabolic activity in the tumour mass despite three courses of adjuvant chemotherapy and metabolically active metastases in the skeleton, not visible on previously scheduled imaging studies.

\section{Conclusion}

The PET-CT study with 18FDG could be of great value for angiosarcoma staging and predicting of the metabolic response to the applied chemotherapy regimen. 


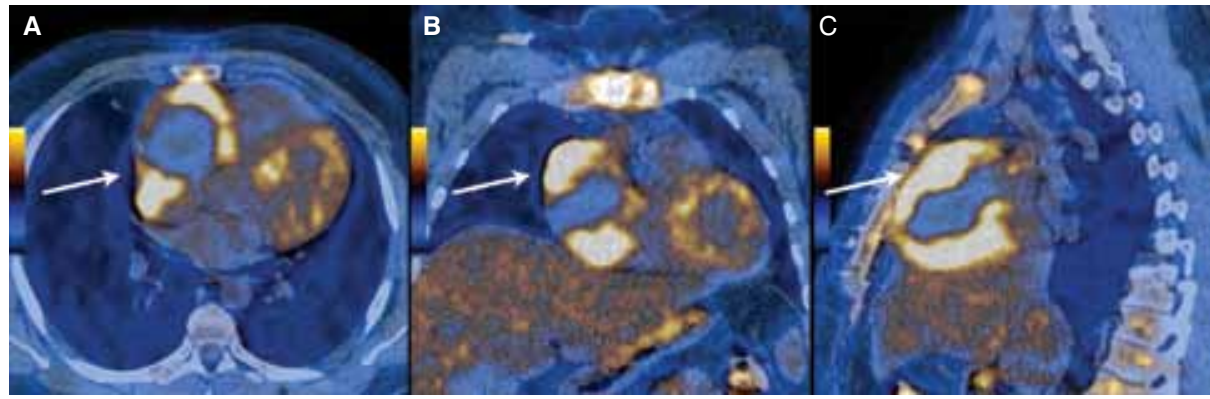

Figure 1. The 18FDG PET-CT study of a patient with cardiac angiosarcoma. A. Axial; B. Coronal; C. Sagittal slices

A

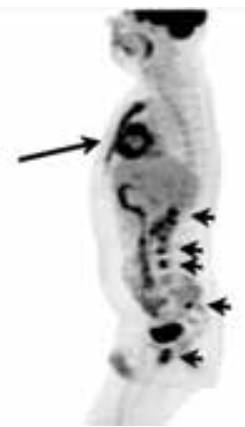

B
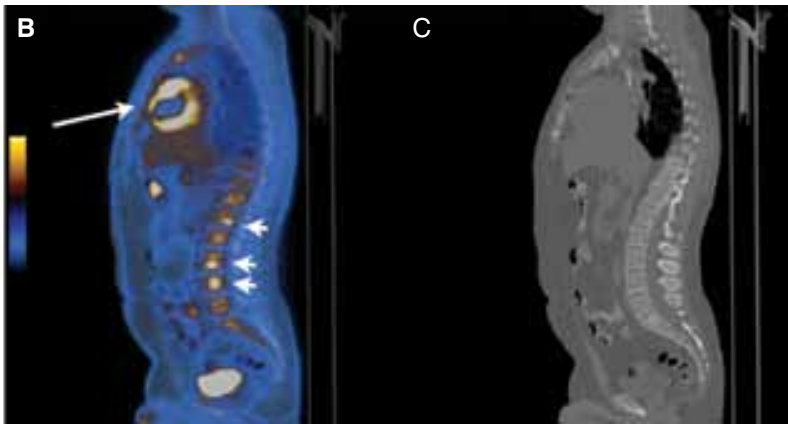

Figure 2. The 18FDG PET-CT study of a patient with cardiac angiosarcoma depicting both heart and skeletal involvement. A. MIP lateral projection; B. Combined PET-CT studies; C. CT study

\section{References}

1. Young, RJ, Brown NJ, Reed MW, Hughes D, Woll PJ. Angiosarcoma. Lancet Oncol 2010; 11: 983-991.

2. Andersen NJ, Froman RE, Kitchell BE, Duesbery NS. Clinical and Molecular Biology of Angiosarcoma. Soft Tissue Tumors 2011; ISBN 978-953-307-862-5.

3. Kakizaki S, Takagi H, Hosaka Y. Cardiac angiosarcoma responding to multidisciplinary treatment. Int J Cardiol 1997; 62: 273-275.

4. Beyer T, Antoch G, Muller S et al. Acquisition Protocol Considerations for Combined PET/CT Imaging. J Nucl Med 2004; 45: 25S-35S.
5. Higashiyama S, Kawabe J, Hayashi T et al. Effectiveness of preoperative PET examination of huge angiosarcoma of the heart. Clin Nucl Med. 2009; 34: 99-102.

6. Juergens KU, Hoffmeier A, Riemann B, Mainz D. Early detection of local tumour recurrence and pulmonary metastasis in cardiac angiosarcoma with PET-CT and MRI. Eur Heart J 2007; 28: 663.

7. Hori Y, Funabashi N, Miyauchi $\mathrm{H}$ et al. Angiosarcoma in the right atria demonstrated by fusion images of multislice computed tomography and positron emission tomography using F-18 Fluoro-Deoxyglucose. Int J Cardiol 2007; 123: e15-e17.

8. Ak I, Ciftci OD, Ustunel Z, Sivrikoz MC. Atrial angiosarcoma imaged by F-18 FDG PET/CT. Anadolu Kardiyol Derg 2011; 11: E17. 